\title{
Alterações na composição corporal e em parâmetros antropométricos de dependentes de crack internados em unidade de adição
}

\author{
Changes in body composition and anthropometric parameters \\ in crack-cocaine addicts admitted in an addiction unit
}

Fernanda Franz Willhelmn ${ }^{1,2}$, Mariana Escobar ${ }^{2,3}$, Ingrid D. Schweigert Perry ${ }^{1,2}$

\section{RESUMO}

Objetivos: Verificar as alterações da composição corporal e de parâmetros antropométricos de dependentes de crack internados para tratamento da adição. Métodos: Estudo de coorte prospectivo, com 40 voluntários masculinos dependentes de crack, de 18 a 60 anos, em tratamento. Foram aferidos parâmetros antropométricos e de composição corporal, por meio de bioimpedância elétrica, na internação e alta hospitalar. Resultados: Com idade média de 29,3 $\pm 6,9$ anos, os pacientes tiveram, durante a abstinência, aumento de peso de 7,6 \pm $3,7 \mathrm{~kg} ; 11,6 \pm 6,4 \%$ do peso corporal; $5,6 \pm 4,2 \mathrm{~cm}$ de circunferência de cintura. Houve aumento de 4,2 $\pm 3,2 \mathrm{~kg}$ de gordura, 3,5 $\pm 3,0 \mathrm{~kg}$ de massa magra e de 2,5 $\pm 2,6$ litros de água. Ao se internarem, $75 \%$ estavam eutróficos, $17,5 \%$ apresentavam sobrepeso e $5 \%$ apresentavam desnutrição, valores que, na alta, se alteraram para $50 \%$ de eutrofia e $47,6 \%$ de sobrepeso (IMC - Índice de Massa Corporal). Observou-se que a média de ganho de peso foi maior nas duas

\section{Palavras-chave}

Crack, antropometria, composição corporal.

primeiras semanas de internação. Conclusão: Ao longo da internação, foram identificados ganho de peso e alterações de composição corporal e nos parâmetros antropométricos dos pacientes, refletindo em migração da eutrofia para o sobrepeso em parcela expressiva deles.

\begin{abstract}
Objectives: Verify the alterations of body composition and of anthropometric parameters in crack addicted patients, hospitalized for addiction treatment. Methods: Cohort prospective studies, with 40 crack addicted male volunteers, 18 to 60 years old, in treatment. Anthropometric parameters and body composition were measured, through bioelectric impedance analysis, within the hospital and at discharge. Results: With an average age $29.3 \pm 6.9$ years, the patients have had, during abstinence, a weight gain of $7.6 \pm 3.7 \mathrm{~kg} ; 11.6 \pm 6.4 \%$ of body weight; $5.6 \pm 4.2 \mathrm{~cm}$ of waist circumference. There was an increase of $4.2 \pm 3.2 \mathrm{~kg}$ of fat (obesity); $3.5 \pm 3.0 \mathrm{~kg}$ of lean body mass and $2.5 \pm 2.6$ liters of water. When hospitalized, $17.5 \%$ of the patients presented overweight and $5 \%$ were malnourished, values that, at discharge, altered to $50 \%$ of eutrophy and $47.6 \%$ of overweight (BMI - Body Mass Index). It was observed that the average of weight gain increased in the first two weeks of hospitalization. Conclusion:
\end{abstract}

1 Hospital de Clínicas de Porto Alegre (HCPA), Residência Integrada Multiprofissional em Saúde. 2 Universidade Federal do Rio Grande do Sul (UFRGS), HCPA, Centro de Estudos em Alimentação e Nutrição. 3 HCPA, Serviço de Nutrição e Dietética. 


\section{Keywords}

Crack, anthropometry, body composition.
During hospitalization a weight gain was identified as well as alterations in body composition and in anthropometric parameters of patients, resulting in migration from eutrophy to overweight, in an expressive group.

\section{INTRODUÇÃO}

O consumo de cocaína é amplo, crescente e preocupante, constituindo-se um dos maiores problemas de saúde pública e abrangendo uma camada da população potencialmente produtiva'. Derivado da cocaína, o crack vem assumindo posição de destaque em virtude do aumento mundial do seu consumo nas últimas décadas ${ }^{2,3}$.

No Brasil, o número de usuários, estimado pelos últimos levantamentos nacionais, aumentou de 0,4\% em $2001^{4}$ para 1,4\% em 2012 5 . Os serviços ambulatoriais especializados para tratamento da dependência começaram a sentir o impacto desse crescimento a partir dos anos 1990, quando em alguns centros a proporção de usuários de crack foi de 17\% (1990) para 64\% (1994) 6 .

O crack é resultado da mistura entre bicarbonato de sódio, adulterantes e cloridrato de cocaína (formando a "base livre") e, quando fumado, produz pequenas partículas absorvidas pelo pulmão, causando a sensação de prazer fugaz, o que pode ser um dos fatores responsáveis pelo início do abuso e da dependência da droga ${ }^{7}$. Seus efeitos no organismo, porém, são mais pronunciados comparados à utilização de outras vias por causa da liberação da cocaína diretamente na corrente sanguínea, por meio dos pulmões. A dependência ao crack acarreta rapidamente deterioração da vida mental, orgânica e social do indivíduo ${ }^{8}$. A intoxicação aguda pode produzir quadro de anorexia e trazer comprometimento do sistema digestivo, sendo comum o aparecimento de náuseas, vômitos e diarreia e, em alguns casos mais graves, o surgimento de úlceras no trato gastrointestinal'.

A avaliação da composição corporal tem recebido importância cada vez maior por causa do papel dos componentes corporais na saúde humana. O índice de massa corporal (IMC) é um dos indicadores mais utilizados na avaliação do estado nutricional, entretanto não é capaz de fornecer informações sobre a distribuição de gordura e composição corporal. A circunferência da cintura (CC) permite avaliar e predizer a gordura corporal, sendo, contudo, uma medida qualitativa, e não quantitativa ${ }^{10}$.

Já a análise da composição corporal por meio da bioimpedância elétrica (BIA) fornece os valores de massa de gordura, massa magra e água corporal por meio de equações preditivas ajustadas para sexo, idade, peso, altura e nível de atividade física"1". A BIA é baseada na condução de uma corrente elétrica indolor de baixa intensidade aplicada ao organismo por meio de cabos conectados a eletrodos ou superfícies condutoras, que são colocados em contato com a pele. Fundamenta-se no princípio de que os tecidos corporais ofe- recem diferentes oposições à passagem da corrente elétrica. Essa oposição, chamada de impedância (Z), tem dois vetores, denominados resistência $(R)$ e reactância $(X c)$. A impedância é baixa no tecido magro, onde se encontram, principalmente, os líquidos e eletrólitos, e alta no tecido adiposo' ${ }^{12}$.

Apesar de conhecidas as diversas consequências clínicas do uso de $c r a c k^{1,13}$, poucos estudos avaliaram a associação da composição corporal e do estado nutricional com o uso de substâncias psicoativas ${ }^{14}$. Da mesma forma, os efeitos de programas de desintoxicação sobre essas variáveis foram pouco explorados ${ }^{15}$, evidenciando lacuna de informações quanto a esses aspectos, o que motivou o presente estudo. Este foi, portanto, delineado com o objetivo de verificar as alterações da composição corporal e de parâmetros antropométricos de dependentes de crack internados em uma unidade de tratamento da adição.

\section{MÉTODOS}

Trata-se de estudo de coorte prospectivo, com amostra de conveniência composta por voluntários do sexo masculino, internados para desintoxicação no período de março a agosto de 2012, na Unidade de Adição do Hospital de Clínicas de Porto Alegre (HCPA), diagnosticados como dependentes de cocaína fumada/crack segundo critérios do Manual de Diagnóstico e Estatística de Transtornos Mentais ${ }^{16}$.

A unidade foi inaugurada em março de 2012 e conta com 20 leitos masculinos atendidos por uma equipe multiprofissional. O objetivo geral da unidade de adição é realizar o tratamento e a reinserção social de indivíduos com transtornos relacionados à adição de drogas, promovendo melhora clínica/ psiquiátrica e redução dos danos, em articulação com a rede de atenção em saúde. O tratamento é dividido em três etapas. A primeira etapa é a de desintoxicação, e nela são analisados os diagnósticos de comorbidades clínicas. A segunda etapa é a de reabilitação focada na motivação e adesão ao tratamento, na qual são trabalhados o manejo da fissura e prevenção de recaída. A terceira e última etapa é a de preparação para alta e reinserção, e nela são realizadas tarefas tais como prevenção de recaída, manutenção de motivação, programas de reinserção e planejamento da alta. Além de consultas individuais, o tratamento tem base nas atividades grupais. O programa conta com atividades físicas diárias, incluindo atividades aeróbicas e de força. A alimentação ocorre no refeitório e é dividida em seis refeições diárias, e no almoço e no jantar os pacientes se servem conforme sua tolerância e aceitação, sendo, portanto, imprecisa a análise do seu consumo alimentar. 
O crack deveria ser a droga de preferência dos participantes, não sendo critério de exclusão o uso de outras substâncias psicoativas. Os pacientes deveriam ter idade entre $18 \mathrm{e}$ 60 anos e ser clinicamente estáveis e com período de internação maior ou igual a 15 dias.

Os critérios de exclusão utilizados foram: a impossibilidade de aplicação da BIA (portadores de marca-passo, amputação de membro inferior e ou superior inteiro, impossibilitando a aplicação dos eletrodos ou integridade de pele prejudicada) e inviabilidade de avaliação da composição corporal e antropométrica dentro das 72 horas pós-baixa e pré-alta.

Foram avaliados parâmetros antropométricos [peso, estatura, CC, circunferência do braço (CB) e dobra cutânea tricipital (DCT)] e composição corporal, além da idade e aspectos clínicos (consumo de substâncias psicoativas, tempo de abstinência, comorbidades e medicação utilizada).

Os dados de idade e clínicos foram obtidos do prontuário dos pacientes. A avaliação antropométrica foi realizada nas primeiras 72 horas de internação. Foram verificados peso e estatura com a utilização de balança Líder ${ }^{\circledR}$ LD 1050 (Líder, Brasil) com precisão de $50 \mathrm{~g}$ com estadiômetro acoplado, para determinação do IMC (peso em m/estatura em $\mathrm{cm}^{2}$ ). $\mathrm{O}$ estado nutricional foi classificado de acordo com os pontos de corte da Organização Mundial da Saúde ${ }^{17}$. As CB e CC foram avaliadas utilizando-se fita métrica com sensibilidade de 1 mm (Mercur ${ }^{\circledast}$, Brasil), e para a mensuração da DCT utilizouse adipômetro de marca Cescorf Científico (Cescorf ${ }^{\oplus}$, Brasil), com capacidade de avaliar até $100 \mathrm{~mm}$ e sensibilidade de $0,1 \mathrm{~mm}$, seguindo protocolo de Duarte ${ }^{18}$. Semanalmente, os pacientes foram pesados na mesma balança e seguindo o mesmo protocolo ${ }^{18}$.

A composição corporal, igualmente avaliada 72 horas após a internação, foi analisada por meio do aparelho de BIA Maltron BF 906 (Maltron ${ }^{\oplus}$, Reino Unido), com os pacientes em jejum de 2 a 3 horas, 30 minutos após a eliminação da urina, sem prática de exercícios físicos nas 12 horas que antecederam o teste, seguindo as instruções pré-teste descritas pelo fabricante. O método tetrapolar utiliza quatro eletrodos aplicados na mão, no punho, no pé e no tornozelo direitos. Esse aparelho fornece dados de massa magra (MM), massa gorda (MG) e água corporal total. O procedimento com a BIA e a avaliação antropométrica foi repetido dentro das 72 horas prévias à alta hospitalar.

Para análise dos dados, foi utilizado o software Statistical Package for the Social Sciences, versão 18.0 (SPSS Inc., Chicago, IL). A análise exploratória dos dados contou com testes descritivos e de frequência. A análise inferencial empregou o teste $t$ de Student para amostras pareadas e o teste de Wilcoxon para variáveis não paramétricas. Foram verificadas associações entre a evolução dos parâmetros antropométricos e de composição corporal com tempo de abstinência por meio do coeficiente de correlação linear de Spearman. Para avaliar a proporção de pacientes nas diferentes classificações do esta- do nutricional na internação e na alta hospitalar, foi utilizado o teste de McNemar. Para avaliar a diferença do ganho de peso semanal, foi utilizado o Equações de Estimação Generalizadas (GEE/Bonferroni). O nível de significância considerado foi 5\%.

O estudo foi aprovado por Comitê de Ética em Pesquisa do HCPA (protocolo no 120.060). Os dados foram coletados depois de cada indivíduo ser informado sobre os procedimentos e objetivos e ter consentido em participar do estudo por meio da assinatura do Termo de Consentimento Livre e Esclarecido. Adicionalmente, os pesquisadores assinaram o Termo de Compromisso para Uso de Dados.

\section{RESULTADOS}

A amostra foi composta por 40 homens, com idade média de 29,3 $\pm 6,9$ anos. O tratamento teve duração média de 30,5 $\pm 7,1$ dias. O perfil dos pacientes quanto a comorbidades clínicas e medicação utilizada durante o período de desintoxicação pode ser visto na tabela 1 .

Tabela 1. Perfil dos pacientes dependentes de crack internados para tratamento $(n=40)$ no Hospital de Clínicas de Porto Alegre entre março e agosto de 2012

\begin{tabular}{lc}
\hline Variável & $\mathbf{N}(\%)$ \\
\hline Comorbidades clínicas & \\
Hipertensão arterial & $4(10,0)$ \\
HIV & $3(7,5)$ \\
Síflis & $2(5,0)$ \\
Tuberculose & $1(2,5)$ \\
Diabetes melito & $1(2,5)$ \\
Medicamento em uso & \\
Benzodiazepínicos & \\
Clonazepam & $33(82,5)$ \\
Diazepam & $18(45,0)$ \\
Lorazepam & $13(32,5)$ \\
Antidepressivos & $2(5,0)$ \\
Metilfenidato & $5(12,5)$ \\
Fluoxetina & $4(10,0)$ \\
Sertralina & $1(2,5)$ \\
Amitriptilina & $1(2,5)$ \\
Antipsicóticos & $1(2,5)$ \\
Clorpromazina & $40(100)$ \\
Risperidona & $34(85,0)$ \\
Olanzapina & $5(12,5)$ \\
Haloperidol & $1(2,5)$ \\
Anticonvulsivantes & $1(2,5)$ \\
Ácido valproico & $17(42,5)$ \\
Carbamaziramato & $14(35,0)$ \\
\hline & $2(5,0)$ \\
\hline & $1(2,5)$ \\
\hline
\end{tabular}


O percentual de dependentes que fizeram uso ao longo da vida e a idade de início do uso das substâncias psicoativas estão representados na tabela 2 , podendo-se observar uma média de idade de início do uso do crack de 21,5 $\pm 6,1$ anos.

Durante a abstinência, os pacientes tiveram aumento de peso de, em média, 7,6 $\pm 3,7 \mathrm{~kg}$; de 11,6 $\pm 6,4 \%$ do peso corporal; de 2,5 $\pm 1,3 \mathrm{~kg} / \mathrm{m}^{2}$ de IMC; de 5,6 $\pm 4,2 \mathrm{~cm}$ de CC; de $2,2 \pm 1,5 \mathrm{~cm}$ de $C B$ e de $6,1 \pm 4,5 \mathrm{~mm}$ de DCT. Já em relação à composição corporal, houve aumento do percentual de gordura de 3,7 $\pm 3,2 \%$ (representando ganho de, em média, 4,2 $\pm 3,2 \mathrm{~kg}$ de $\mathrm{MG}$ ) e redução proporcional do percentual MM de $3,6 \pm 3,2 \%$ e de água $(2,7 \pm 2,3 \%)$, representando, contudo, ganho de 3,5 $\pm 3,0 \mathrm{~kg}$ de MM e de 2,5 \pm 2,6 litros para a quantidade de água.

Os valores das variáveis antropométricas e de composição corporal podem ser vistos na tabela 3.
Considerando a classificação do estado nutricional, foram verificadas mudanças no percentual de pacientes eutróficos e com excesso de peso ao longo do período de tratamento, como pode ser visto na figura 1.

A figura 2 representa a evolução de ganho de peso durante a internação, observando-se diferenças estatisticamente significativas entre as duas primeiras semanas de internação e as duas últimas, porém não houve diferença significativa entre o ganho de peso das duas primeiras semanas nem entre as duas últimas entre si. O mesmo padrão ocorreu com o percentual de ganho de peso, que foi de 5,0 $\pm 0,6 \%$ na primeira semana; de 3,1 $\pm 0,5 \%$ na segunda semana; de 1,5 \pm $0,4 \%$ na terceira semana; e de 1,5 $\pm 0,3 \%$ na quarta semana $(p<0,01$ para semana $1 \times$ semana 3, semana $1 \times$ semana 4 , $p<0,01$; semana $2 \times$ semana $4 ; p=0,02$ para semana $2 \times$ semana 3, $p=0,02$, teste de GEE/Bonferroni), sem significância entre as duas primeiras semanas e as duas últimas.

Tabela 2. Percentual de dependentes que fizeram uso ao longo da vida e idade do primeiro uso das substâncias psicoativas pelos pacientes dependentes de crack internados para tratamento (Hospital de Clínicas de Porto Alegre, entre março e agosto de 2012)

\begin{tabular}{lcc}
\hline Substâncias psicoativas & $\begin{array}{c}\text { Usuários que fizeram uso ao longo da vida } \\
\text { Percentual (\%) }\end{array}$ & $\begin{array}{c}\text { Idade de início de uso em anos } \\
\text { Média } \pm \text { desvio-padrão }\end{array}$ \\
\hline Álcool & 85,0 & $12,7 \pm 2,8$ \\
Tabaco & 87,5 & $14,6 \pm 3,8$ \\
Maconha & 90,0 & $15,1 \pm 2,1$ \\
Inalantes & 42,5 & $15,4 \pm 3,8$ \\
Cocaína aspirada & 77,5 & $17,7 \pm 3,9$ \\
Ecstasy & 7,5 & $19,7 \pm 2,1$ \\
Pitico (maconha com crack) & 47,5 & $21,1 \pm 5,6$ \\
Crack & 100,0 & $21,5 \pm 6,1$ \\
LSD & 7,5 & $22,7 \pm 3,2$ \\
Oxi & 2,5 & $24,0 \pm 4,2$ \\
\hline
\end{tabular}

Tabela 3. Composição corporal e parâmetros antropométricos dos pacientes dependentes de crack internados para tratamento (Hospital de Clínicas de Porto Alegre, entre março e agosto de 2012)

\begin{tabular}{|c|c|c|c|}
\hline Variável & Na internação hospitalar* & Na alta hospitalar* & p \\
\hline Peso $(\mathrm{kg})^{\mathrm{a}}$ & $66,10(61,92-74,75)$ & $74,50(69,65-82,47)$ & $<0,001$ \\
\hline $\operatorname{IMC}\left(\mathrm{kg} / \mathrm{m}^{2}\right)^{\mathrm{a}}$ & $22,44(20,77-24,28)$ & $24,90(22,92-26,52)$ & $<0,001$ \\
\hline Circunferência de cintura $(\mathrm{cm})^{\mathrm{a}}$ & $80,95(76,72-86,75)$ & $87,45(83,85-92,00)$ & $<0,001$ \\
\hline Dobra cutânea tricipital (mm) ${ }^{\mathrm{a}}$ & $8,15(6,07-13,17)$ & $15,13(9,12-21,8)$ & $<0,001$ \\
\hline Circunferência de braço $(\mathrm{cm})^{b}$ & $28,00 \pm 2,74$ & $30,21 \pm 2,48$ & $<0,001$ \\
\hline Percentual de gordura $(\%)^{b}$ & $16,48 \pm 5,73$ & $20,15 \pm 4,96$ & $<0,001$ \\
\hline Percentual de massa magra $(\%)^{b}$ & $83,48 \pm 5,72$ & $79,83 \pm 4,95$ & $<0,001$ \\
\hline Peso de massa gorda $(\mathrm{kg})^{\mathrm{a}}$ & $10,20(7,72-14,42)$ & $14,10(10,50-19,32)$ & 0,002 \\
\hline Peso de massa magra $(\mathrm{kg})^{\mathrm{a}}$ & $57,10(52,08-59,58)$ & $60,35(55,70-64,38)$ & $<0,001$ \\
\hline Água (L) ${ }^{\mathrm{a}}$ & $41,80(38,03-43,60)$ & $44,15(40,80-47,10)$ & $<0,001$ \\
\hline Percentual de água $(\%)^{b}$ & $61,10 \pm 4,14$ & $58,48 \pm 3,61$ & $<0,001$ \\
\hline
\end{tabular}

* Média (desvio-padrão) ou mediana (P25-P75).

'Teste de Wilcoxon

${ }^{\mathrm{b}}$ Teste $\mathrm{t}$ de Student pareado. 


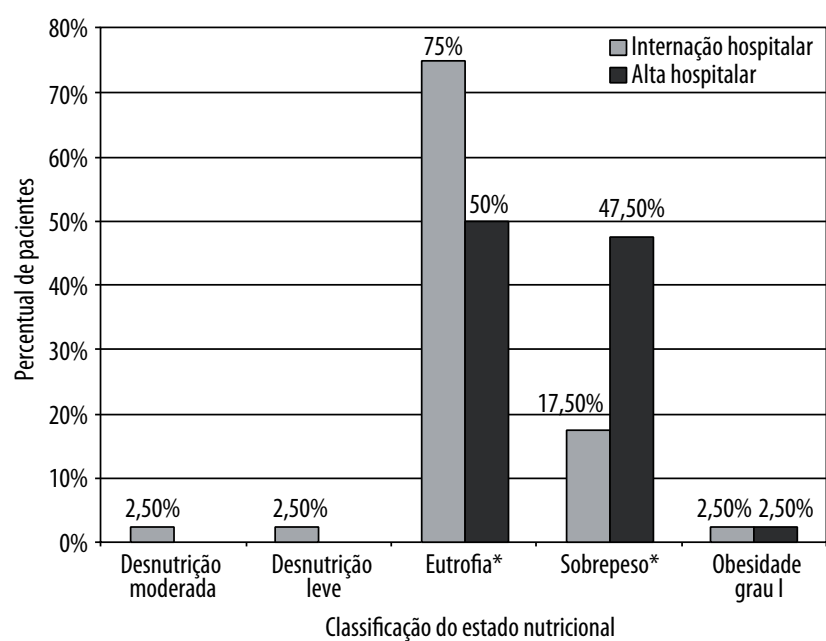

Figura 1. Alterações na classificação nutricional dos pacientes dependentes de crack no momento da internação e da alta hospitalar (Hospital de Clínicas de Porto Alegre, 2012). Barras representam o percentual de pacientes em cada classificação do estado nutricional $(n=40)$. * Representa percentuais estatisticamente diferentes entre os dois momentos de avaliação (McNemar; $p<0,05$ ).

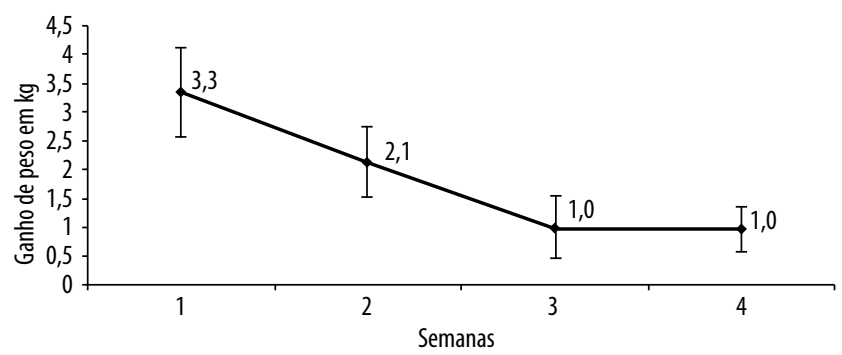

Figura 2. Ganho de peso (kg) semanal dos dependentes de crack internados (Hospital de Clínicas, 2012), $p<0,01$ para semana $1 \times$ semana 3 , semana $1 \times$ semana 4 , semana $2 \times$ semana $3 ; p=0,02$ para semana $2 \times$ semana 4 (GEE/Bonferroni).

Ainda considerando o tempo de internação, foram analisadas as correlações entre este e as variáveis antropométricas e de composição corporal, não havendo sido observada nenhuma correlação significativa. Apenas a variação de peso mostrou tendência a uma fraca correlação positiva ( $r$ s = 0,294; $p=0,06$; coeficiente de Spearman).

\section{DISCUSSÃO}

A amostra do presente estudo apresenta características semelhantes ao que é referido pela literatura quanto à idade de início de consumo de crack e da ordem da sequência de uso (em anos) de substâncias psicoativas ${ }^{15,19}$. Ainda segundo a literatura, o consumo de substâncias psicoativas por dependentes de crack inicia-se pelas drogas lícitas, sendo a maconha a mais comum da segunda fase de experimenta- ção ${ }^{20}$. Dunn et al. ${ }^{21}$ encontraram em sua pesquisa que quase a totalidade (96\%) dos usuários de crack já havia feito uso ao longo da vida de maconha, sendo álcool (88\%) e tabaco (87\%) também muito prevalentes, com tendência e valores similares aos encontrados neste estudo.

Há poucos dados da literatura a respeito de comorbidades encontradas nesses pacientes, sendo mais abordadas as doenças infecciosas e sexualmente transmissíveis do que doenças crônicas não transmissíveis ${ }^{22}$. Estudo nacional de revisão apontou que o consumo de crack é associado à infecção por HIV e comportamento de risco mais frequente ${ }^{2}$. Azevedo et al. ${ }^{23}$ e Carvalho e Seibe ${ }^{24}$ avaliaram usuários de crack do sexo masculino e detectaram valores de prevalência de HIV de $11 \%$ e $6,6 \%$, respectivamente. O presente estudo encontrou prevalência maior de doenças infecciosas e de transmissão sexual, com percentual de HIV intermediário entre os dois estudos citados.

Durante o período de internação, houve alterações significativas nos parâmetros antropométricos e na composição corporal, em especial no aumento do percentual de gordura e MG, o que repercutiu no aumento de peso dos usuários. Isso não se apresentou, na grande parte dos casos, como algo saudável, e sim como fator de risco para doenças crônicas não transmissíveis, principalmente para diabetes, hipertensão arterial, hipercolesterolemia, hipertrigliceride$\mathrm{mia}^{10}$, uma vez que se refletiu em condições de sobrepeso. Balbinot et al. ${ }^{14}$ também observaram alterações corporais importantes durante o período de desintoxicação, com aumento do peso de gordura, entretanto não de peso de MM. No presente estudo, o aumento significativo de MM, possivelmente, se mostrou principalmente em ganho de água, e não em ganho de massa muscular, o que pode repercutir diretamente nas valências físicas dos indivíduos, em suas capacidades funcionais, e pode influenciar no surgimento e/ou agravamento tanto de doenças metabólicas quanto psíqui$\mathrm{cas}^{25}$. Vale ressaltar que o programa de tratamento do local do estudo inclui atividades físicas aeróbicas e anaeróbicas, que também podem ter contribuído para o aumento de MM.

Apesar de, no presente estudo, ser restrito o número de pacientes com alterações cardiovasculares e metabólicas, sabe-se que, além dos efeitos nocivos do excesso de peso para o sistema cardiovascular, esse sistema também é alvo das complicações do crack mais comprometedoras e potencialmente ameaçadoras à vida do usuário, como angina, infarto agudo do miocárdio e aumento do volume cardíaco, acidentes vasculares cerebrais e arritmias cardíacas. A cocaína e seus derivados podem causar aumento da pressão arterial e da frequência cardíaca de maneira dose-dependente. Os efeitos potencializadores da droga sobre o sistema cardiovascular são decorrentes da ação antagonista central sobre as monoaminas, podendo deixar o organismo mais sensível à ação destas, aumentar a frequência cardíaca, a pressão arterial, a contração cardíaca e o potencial de relaxamento 
cardíaco e vasoconstrição coronariana. Estudo indica que o risco de infarto agudo do miocárdio até 60 minutos após o uso de cocaína é 24 vezes maior em pacientes de baixo risco. A aterosclerose coronária prematura tem sido vista em 35\% a 40\% dos dependentes jovens de cocaína. Além disso, a cocaína pode também aumentar a agregação plaquetária por mecanismo alfa-adrenérgico e potencializar a produção de tromboxano pelas plaquetas ${ }^{26}$.

É importante ressaltar que os pacientes, ao se internarem, na sua maioria apresentavam estado nutricional adequado (75\%), seguido de sobrepeso (17,5\%) pelos critérios do IMC. Apenas 5,0\% encontravam-se em algum grau de desnutrição. Esses aspectos podem estar ligados ao fato de que a amostra era composta de pacientes que se internaram voluntariamente para tratamento. Sabe-se que apenas 10\% dos usuários de cocaína procuram tratamento ${ }^{5}$, podendo, assim, os participantes desta pesquisa possuir características clínicas e estado físico mais preservados que os demais usuários ativos da droga, refletindo no seu estado nutricional e de composição corporal. Esse fato também pode estar relacionado com o prognóstico de alterações corporais desses pacientes.

Em virtude do ganho de peso na internação, o estado nutricional dos usuários deste estudo também se alterou, resultando na diminuição do percentual de eutróficos para $50 \%$ e aumento nos percentuais de sobrepeso para $47,6 \%$, além de migração de classificação do estado nutricional dos desnutridos para a eutrofia. Esses resultados vão de encontro aos da literatura no que se refere a pacientes dependentes químicos em processo de recuperação, em especial em recuperação de cocaína/crack. Oliveira et al. ${ }^{27}$ avaliaram os hábitos alimentares e o perfil antropométrico de dependentes químicos em tratamento em uma fazenda terapêutica e encontraram prevalência maior de eutrofia $(76,92 \%)$ seguida de sobrepeso $(19,23 \%)$ e obesidade $(1,92 \%)$. Pesquisa feita com usuários de múltiplas drogas na Noruega encontrou 83\% de indivíduos com IMC dentro do adequado, 3\% abaixo do normal e $14 \%$ acima do índice esperado ${ }^{28}$.

Pesquisas observaram excesso de peso e padrões alimentares disfuncionais em indivíduos em recuperação da adição de drogas ${ }^{29,30}$, tendo dieta pobre em nutrientes essenciais, com baixo consumo de leite e derivados, de frutas e verduras e, consequentemente, de vitaminas e minerais, fibras e proteína animal ${ }^{31}$. A hiperfagia rebote seguida à abstinência pode ser um mecanismo para restabelecer os neurotransmissores (dopamina e serotonina, principalmente) do sistema de recompensa, levando ao aumento de peso e do IMC durante a recuperação da adição a substâncias ${ }^{32}$. No presente estudo, o fato de o paciente poder servir-se de acordo com a própria tolerância e aceitação em duas das principais refeições pode ter contribuído para maior consumo alimentar.

Reguladores hormonais da homeostase energética podem agir também no sistema de recompensa do cérebro, principalmente no sistema de dopamina do mesoaccumbens $^{33}$, para aumentar ou diminuir o valor de incentivo de alimentos, dependendo das necessidades energéticas. No entanto, a estimulação elétrica ou química das áreas do cérebro que regulam a recompensa alimentar pode provocar compulsão alimentar, uma vez que os sinais de saciedade homeostáticos ficam comprometidos ${ }^{34}$. Isso sugere que a obtenção dos efeitos prazerosos da comida é uma poderosa força motivadora que pode substituir os sinais de saciedade e, sendo assim, alimentos mais palatáveis (com mais açúcares e gorduras) geralmente são consumidos com maior frequência e em maior quantidade. Os sistemas de regulação da ingestão de alimentos e uso de drogas são os mesmos, e respostas adaptativas similares podem acionar o sistema de recompensa no cérebro. Como resultado, a obesidade é agora conceituada como uma forma de comportamento compulsivo, similar ao vício em substâncias psicoativas ${ }^{35}$.

Outro fato que pode estar associado ao ganho de peso desses pacientes é a medicação utilizada para o tratamento de crack, que tem como possíveis efeitos colaterais o aumento de apetite e consequente aumento da ingestão alimentar e ganho de peso ${ }^{36}$.

Embora não tenha havido correlação entre o tempo de internação e as diversas variáveis antropométricas e de composição corporal, possivelmente por causa de certa similaridade do período de permanência dos pacientes, houve maior ganho de peso e, possivelmente, de gordura corporal nas duas primeiras semanas de internação, com considerável diminuição da velocidade de ganho nas semanas seguintes. Estudos com animais e seres humanos evidenciam ganho de peso após períodos de privação ou restrição, similar ao que ocorre com usuários de $\mathrm{crack}^{37}$. O período inicial de recuperação da dependência química mostra ser aquele com ganho de peso mais intenso, podendo ultrapassar inclusive a perda de peso no período de adição ${ }^{38}$. Após privação alimentar, a deficiência de energia pode ser rapidamente restaurada com o início da realimentação ${ }^{39}$, ocorrendo rápido ganho de peso e acúmulo de gordura ${ }^{40}$. Isso ocorre pelo fato de a concentração de leptina ser inversamente proporcional ao apetite ${ }^{41}$, portanto restrições calóricas levam à superalimentação após o período de realimentação ${ }^{42}$, o que promove o aumento da massa gorda ${ }^{43}$. Apesar de essa ser uma explicação plausível para dependentes químicos em tratamento, no presente estudo, pelo estado nutricional prévio, não é possível afirmar que tenha havido privação pelos dados deles, podendo talvez esse fenômeno ser atribuído muito mais à substituição da droga pela alimentação no mecanismo de recompensa.

Este estudo apresentou como limitações o tamanho da amostra, a falta de grupo controle, a utilização concomitante de anticonvulsivantes e antipsicóticos e a polidependência associada a diferentes efeitos fisiológicos e psíquicos. Nesse sentido, a ampliação da amostra, a avaliação do consumo alimentar prévia e durante a internação, assim como 
a busca da associação entre atividade física e mudança da composição corporal, efeitos dos medicamentos e análise de aspectos da polidependência, constituem perspectivas a serem investigadas nessa população. Do acompanhamento ambulatorial pós-alta também poderão derivar informações importantes quanto aos efeitos da abstinência no médio e no longo prazos.

\section{CONCLUSÃO}

Foi possível identificar que, ao longo da internação hospitalar, ocorreram alterações importantes nos parâmetros antropométricos e de composição corporal. Foi identificado ganho de peso, mais intenso nas duas primeiras semanas de tratamento, e alterações de composição corporal e nos parâmetros antropométricos, refletindo em migração da eutrofia para o sobrepeso em parcela expressiva dos pacientes.

Essas alterações não são necessariamente produtivas para saúde geral e qualidade de vida do dependente químico, levando à reflexão sobre se esses fenômenos não deveriam ser levados em consideração na construção do plano terapêutico para o usuário de crack. O ganho de peso excessivo que geralmente ocorre no início da recuperação realça ainda mais a importância de abordar o tema da alimentação no tratamento para usuários de substâncias psicoativas, incluindo nele metas adequadas para recuperação de peso, planos dietéticos que visem a práticas alimentares saudáveis e oficinas de educação nutricional, além do incentivo à prática de atividades físicas, contemplando mudanças de hábitos que, realizadas com o apoio e a integração de equipe multiprofissional, promovam alterações no estilo de vida e no controle de comportamentos adictos desses pacientes.

Além disso, sabendo que o crack é no momento um dos maiores problemas de saúde pública do país, mais estudos voltados para esses aspectos tornam-se relevantes em vista das implicações que poderão ter sobre alternativas terapêuticas eficazes para o tratamento, minimizando, contudo, impactos sobre o comprometimento de outros aspectos de saúde e qualidade de vida dos dependentes.

\section{CONTRIBUIÇÕES INDIVIDUAIS}

Fernanda Franz Willhelm - Concepção e desenho do estudo, elaboração do artigo e aprovação da versão final a ser publicada.

Mariana Escobar - Análise e interpretação dos dados, revisão crítica do conteúdo intelectual do artigo e aprovação da versão final a ser publicada.

Ingrid D. Schweigert Perry - Concepção e desenho do estudo e elaboração do artigo, revisão crítica do seu conteúdo intelectual e aprovação da versão final a ser publicada.

\section{CONFLITOS DE INTERESSE}

Os autores não possuem conflitos de interesse a serem declarados.

\section{AGRADECIMENTO}

Ao Fundo de Incentivo a Pesquisa e Eventos do Hospital de Clínicas de Porto Alegre/RS, pelo suporte financeiro, e à acadêmica de Nutrição Cristiane Schulz Parizotti (UFRGS), pelo auxílio na coleta de dados do trabalho.

\section{REFERÊNCIAS}

1. Mançano A, Marchiori E, Zanetti G, Escuissato DL, Duarte BC, Apolinário LA. Complicaçōes pulmonares após uso de crack: achados na tomografia computadorizada de alta resolução do tórax. J Bras Pneumol. 2008;34(1):323-7.

2. Duailibi LB, Ribeiro M, Laranjeira R. Profile of cocaine and crack users in Brazil. Cad Saude Publica. 2008;24(4):545-57.

3. Oliveira LG, Nappo SA. Crack-cocaine in Sao Paulo: accessibility, market strategies and ways of use. Rev Psiq Clín. 2008;35(6):212-8

4. Carlini EA, Galduróz JCF, Noto AR, Fonseca AM, Carlini CMA, Oliveira LG, et al. II Levantamento domiciliar sobre 0 uso de drogas psicotrópicas no Brasil: estudo envolvendo as 108 maiores cidades do País - 2005. Braślia: Secretaria Nacional Antidrogas; 2007.

5. Laranjeiras R, Madruga CR, Ribeiro M, Pinsky I, Caetano R, Mitsuhiro S. II Levantamento Nacional de Álcool e Drogas. Uso de Cocaína e Crack no Brasil. Instituto Nacional de Ciência e Tecnologia para Políticas Públicas do Álcool e Outras Drogas; 2012.

6. Dunn J, Laranjeira R, Silveira DX, Formigoni MLOS, Ferri CP. Crack cocaine: an increase in use among patients attending clinics in Sao Paulo: 1990-1993. Subst Use Misuse. 1996;31:51927.

7. Ferri CP, Laranjeira RR, Silveira DX, Dunn J, Formigoni ML. Aumento da procura de tratamento por usuários de crack em dois ambulatórios na cidade de São Paulo nos anos de 1990 a 1993. Rev Assoc Med Bras. 1997;43:25-8.

8. Duailibi LB, Ribeiro M, Laranjeira R. Profile of cocaine and crack users in Brazil. Cad Saude Publica. 2008:24(4):545-57.

9. Lizasoian I, Moro MA, Lorenzo, P. Cocaína: aspectos farmacológicos. Adicciones. 2002;14(1):57-64.

10. Rezende FAC, Rosado LEFPL, Franceschinni SCC, Rosado GP, Ribeiro RCL. Aplicabilidade de índice de massa corporal na avaliação da gordura corporal. Rev Bras Med Esporte. 2010;16(2):90-4

11. Rech CR, Cordeiro BA, Petroski EL, Vasconcelos FAG. Validation of bioelectrical impedance for the prediction of fat-free mass in Brazilian elderly subjects. Arq Bras Endocrinol Metab. 2008;52(7):1163-71.

12. Kyle UG, Bosaeus I, Lorenzo AD, Deurenberg P, Elia M, Gómez JM, et al. Bioelectrical impedance analysis - part I: review of principles and methods. Clin Nutr. 2004;23:1226-46.

13. del Castillo Palma MJ, Querol Gutiérrez JJ, Ramos Díaz M, Anglada Pintado JC. Edema pulmonar e insuficiencia renal en paciente adicto a cocaína (crack). An Med Interna (Madrid). 2003;20(1):216-7.

14. Balbinot AD, Alves GSL, Amaral Junior AF, Araujo RB. Associação entre fissura e perfil antropométrico em dependentes de crack. J Bras Psiquiatr. 2011;60(3):205-9.

15. Balbinot AD, Alves GSL, Amaral Junior AF do, Araujo RB. Perfil antropométrico de dependentes de crack hospitalizados para desintoxicação. Rev HCPA. 2011;31(3):311-7.

16. Manual diagnóstico e estatístico de transtornos mentais - DSM-IV-TR. Trad. Cláudia Dornelles. $4^{\mathrm{a}}$ ed. rev. Porto Alegre: Artmed; 2002.

17. World Health Organization. Obesity: preventing and managing the total epidemic. Report of a WHO Consultation Group. Geneva: WH0; 1997. 
18. Duarte ACG. Avaliação nutricional - aspectos clínicos e laboratoriais. 1a ed. São Paulo: Atheneu; 2007. 41p.

19. Sanchez ZVM, Nappo SA. Sequência de drogas consumidas por usuários de crack e fatores interferentes. Rev Saude Publica. 2002;36(4):420-30

20. Sanchez ZVM, Nappo SA. From the first drug to crack: the sequence of drugs taken in a group of users in the city of São Paulo. Subst Use Misuse. 2007;42(1):177-88.

21. Dunn J, Laranjeira R, Silveira DX, Formigoni MLOS, Ferri CP. Crack cocaine: an increase in use among patients attending clinics in Sao Paulo: 1990-1993. Subst Use Misuse. 1996;31:519-27.

22. Mccoy CB, Lai S, Metsch LR, Messiah SE, Zhao W. Injection drug use and crack cocaine smoking: independent and dual risk behaviors for HIV infection. Ann Epidemiol. 2004;14(8):535-42.

23. Azevedo RCS, Botega NJ, Guimaraes LAM. Crack users sexual behavior and risk of HIV infection. Rev Bras Psquiatr. 2007;29(1):26-30.

24. Carvalho HB, Seibel SD. Crack cocaine use and its relationship with violence and HIV. Clinics. 2009;64(9):857-66.

25. Matsudo SM, Matsudo VKR, Barros Neto TL. Impacto do envelhecimento nas variáveis antropométricas, neuromotoras e metabólicas da aptidão física. Rev Bras Ciênc e Mov. 2000;8(4):21-32.

26. Marat S, Figueredo VM, Lynn Morris D. Cocaine and the heart. Clin Cardiol. 2010;33(5):264-9.

27. Oliveira ERN, Marin IC, Ferruzzi L, Tenório MFS, Trindade E. Avaliação dos hábitos alimentares e dos dados antropométricos de dependentes químicos. Arq Cienc Saude Unipar. 2005;9(2):91-6

28. Sæland M, Haugen M, Eriksen FL, Wandel M, Smehaugen A, Böhmer T, et al. High sugar consumption and poor nutrient intake among drug addicts in 0slo, Norway. Br J Nutr. 2012:105:618-24.

29. Hatcher AS. Weight matters during recovery: unhealthy eating behaviors can impede client progress. Addiction Professional. 2004;2(6):23-4.

30. Jackson TD, Grilo CM. Weight and eating concerns in outpatient men and women being treated for substance abuse. Eat Weight Disord. 2002;7(4):276-83.
31. Ribeiro LA, Sanchez ZM, Nappo SA. Surving crack: a qualitative study of strategies and tactics developed by Brazilian users to deal with the risks associated with drug. BMC Public Health. 2010;10:671-81.

32. Gold MS. From bedside to bench and back again: a 30-year saga. Physio Behav. 2011;104(1):157-61.

33. Kenny PJ. Reward mechanisms in obesity: new insights and future directions. Neuron. 2011;69:664-79.

34. Will MJ, Pratt WE, Kelley AE. Pharmacological characterization of high-fat feeding induced by opioid stimulation of the ventral striatum. Physiol Behav. 2006;89:226-34.

35. Kenny PJ. Common cellular and molecular mechanisms in obesity and drug addiction. Nature Rev Neurosci. 2011;12:638-51.

36. Zimmermann U, Kraus T, Himmerich H, Schuld A, Pollmächer T. Epidemiology, implications and mechanisms underlying drug-induced weight gain in psychiatric patients. J Psychiatr Res. 2003;37(3):193-220.

37. Ouwens MA, Van Strien T, van der Staak CPF. Tendency toward overeating and restraint as predictors of food consumption. Appetite. 2003;40:291-8.

38. Cowain J, Devine C. Food, eating and weight concerns of men in recovery from substance addiction. Appetite. 2008;50:33-42.

39. Duarte F0, Sene-Fiorese M, Cheik NC, Santa-Maria ASL, Aquino Jr AE, Oishi JC, et al. Food restriction and refeeding induces changes in lipid pathways and fat deposition in the adipose and hepatic tissues in rats with diet-induced obesity. Exp Physiol. 2012;97(7):882-94.

40. Dulloo AG, Jacquet J, Montani JP. How dieting makes some fatter: from a perspective of human body composition autoregulation. Proc Nutr Soc. 2012;71:379-89.

41. Mars M, de Graaf C, de Groot CP, van Rossum CT, Kok FJ. Fasting leptin and appetite responses induced by a 4 days 65\% energy restricted diet. Int J 0bes. 2006;30:122-8.

42. Ogawa R, Strader AD, Clegg DJ, Sakai RR, Seeley RJ, Woods SC. Chronic food restriction and reduced dietary fat: risk factors for bouts of overeating. Physiol Behav. 2005;86:578-85.

43. Stanley S, Wynne K, McGowan B, Bloom S. Hormonal regulation of food intake. Physiol Rev. 2005;85:1131-58. 